\title{
Stay-at-Home: The Impact of the COVID-19 Lockdown on Household Functioning and ART Adherence for People Living with HIV in Three Sub-districts of Cape Town, South Africa
}

\author{
Linda S. Campbell ${ }^{1}$ (1) $\cdot$ Caroline Masquillier ${ }^{1} \cdot$ Lucia Knight $^{2,3} \cdot$ Anton Delport $^{3} \cdot$ Neo Sematlane $^{1,3}$. \\ Lorraine Tanyaradzwa Dube ${ }^{3} \cdot$ Edwin Wouters $^{1,4}$
}

Accepted: 22 November 2021 / Published online: 3 January 2022

(c) The Author(s), under exclusive licence to Springer Science+Business Media, LLC, part of Springer Nature 2021

\begin{abstract}
In March 2020, the South African government imposed a lockdown to control COVID-19 transmission. Lockdown may affect people living with HIV's (PLWH) antiretroviral therapy (ART) adherence. Data from a cluster randomised control trial was collected from 152 PLWH in Cape Town sub-districts from October 2019-March 2020 when the lockdown halted collection. Subsequently, 83 PLWH were followed-up in June-July 2020. Random effects models were used to analyse: (1) changes between baseline and follow-up and (2) correlates of adherence during lockdown. At follow-up, there was an increase in the odds of being below the poverty line and the odds of experiencing violence decreased. Measures for well-being, household functioning, stigma and HIV competency improved. Violence, depression, food insecurity, and stigma were associated with poorer ART adherence; higher well-being scores were associated with better adherence. During lockdown, governments need to ensure financial support, access to (mental) health services, and services for those experiencing violence.

Clinical Trial Number: Pan African Clinical Trial Registry, PACTR201906476052236. Registered on 24 June 2019.
\end{abstract}

Keywords COVID-19 $\cdot$ HIV $\cdot$ ART $\cdot$ Adherence $\cdot$ Lockdown

\section{Resumen}

En marzo de 2020, el gobierno sudafricano impuso un confinamiento generalizado para controlar la transmisión del COVID19. Estas medidas pueden afectar la adherencia a la terapia antirretroviral de las personas con VIH. A partir de un ensayo aleatorio grupal se recopilan datos de 152 personas con VIH en los sub-distritos de Ciudad del Cabo entre octubre de 2019 y marzo de 2020 cuando el confinamiento detuvo la recopilación. Posteriormente, se realizó un seguimiento de 83 pacientes entre junio y julio de 2020. Se utilizaron modelos de efectos aleatorios para analizar: 1) los cambios en las variables entre la línea de base y de seguimiento; 2) covariables de adhesión al tratamiento. En el seguimiento se observa que aumentaron las probabilidades de estar por debajo del umbral de pobreza y disminuyeron las probabilidades de sufrir violencia. Las medidas de bienestar, funcionamiento del hogar, estigma e idoneidad en materia de VIH mejoraron entre la línea base y el seguimiento. Por otro lado, la violencia, la depresión, la inseguridad alimentaria y el estigma se asocian a una menor

Linda S. Campbell

linda.campbell@uantwerpen.be

Caroline Masquillier

caroline.masquillier@uantwerpen.be

Lucia Knight

lucia.knight@uct.ac.za

Anton Delport

adelport@uwc.ac.za

Neo Sematlane

3879990@myuwc.ac.za

Lorraine Tanyaradzwa Dube

ldube@uwc.ac.za
Edwin Wouters

edwin.wouters@uantwerpen.be

1 Centre for Population, Family and Health, University of Antwerp, Prinsstraat 13, 2000 Antwerpen, Belgium

2 Division of Social and Behavioural Sciences, School of Public Health and Family Medicine, Faculty of Health Sciences, University of Cape Town, Cape Town, South Africa

3 School of Public Health, Community and Health Sciences, University of the Western Cape, Cape Town, South Africa

4 Centre for Health Systems Research \& Development, University of the Free State, Bloemfontein, South Africa 
adherencia al tratamiento antirretroviral, mientras la mejora del bienestar se asocia a una mayor adherencia al tratamiento. De tal manera, se observa que durante situaciones de emergencia que requieran de confinamientos es recomendable que los gobiernos garanticen apoyo financiero, atención a la salud física y mental de manera continua, así como servicios especializados hacia quienes sufren violencia.

Palabras clave COVID-19 · VIH · adhesión · tratamiento antiretroviral $\cdot$ confinamiento

$\begin{array}{ll}\text { Abbreviations } & \\ \text { ART } & \text { Anti-retroviral therapy } \\ \text { CHW } & \text { Community health worker } \\ \text { DHS } & \text { Demographic and health survey } \\ \text { PLWH } & \text { People living with HIV } \\ \text { CI } & \text { Confidence interval } \\ \text { HIV } & \text { Human immunodeficiency virus } \\ \text { LMIC } & \text { Low and middle-income countries } \\ \text { NIDS-CRAM } & \text { National Income Dynamics Study-Coro- } \\ & \text { navirus Rapid Mobile Survey } \\ \text { WHO } & \text { World Health Organisation }\end{array}$

\section{Introduction}

Following the emergence of the novel coronavirus SARSCOV-2 in December 2019 in Wuhan, China, governments and health agencies throughout the world began preparations as the virus spread $[1,2]$. The impact was anticipated to fall most heavily on vulnerable groups in under-resourced countries [3, 4]: fragile health systems, populations living in poverty in densely-populated areas, and a lack of essential infrastructure such as water and sanitation create unique difficulties for the implementation of physical distancing regulations [5]. Governments were faced with the dilemma of protecting their populations from COVID-19, whilst recognizing that lockdown measures may also exacerbate health inequalities and negatively impact health and wellbeing - and may not be effective in the poorest settings [4, 6]. Measures such as lockdown in low and middle-income countries (LMICs) are therefore contentious [4, 6, 7].

Following confirmation of the first case of COVID-19 in South Africa on 5th March 2020 [8], President Cyril Ramaphosa declared a national state of emergency on 15th March [9]. This was shortly followed by a national lockdown on 27th March [10], known as one of the strictest in the world. The lockdown included a stay-at-home order except for exercise within $5 \mathrm{~km}$ of one's home, with visitors and gatherings banned and travel on public transport only permitted at $25 \%$ capacity for essential workers or essential reasons, as well as a ban on alcohol sales [11, 12]. The lockdown remained until 1st May when South Africa moved to level four of a five-level system; travel (for work or education) was once again permitted and certain venues were allowed to reopen with outdoor seating [10]. Health care facilities, a designated essential service, remained open throughout the lockdown [13].

The health implications of COVID-19 coinfection for people living with HIV (PLWH) were not well understood in early 2020. There was therefore alarm about a virus that was known to have adverse outcomes for those with comorbidities $[14,15]$. In South Africa, HIV prevalence was estimated at $14 \%$ in 2017 among the general population of all ages [16] and over half the population still lives in poverty [17]. A study conducted from March to June 2020 using data from the Western Cape furthermore indicated an association between HIV and COVID-19 mortality (aHR 2.14; 95\%CI 1.70-2.70) [18].

A key concern was the potential impact of lockdown regulations on PLWH in terms of adherence to antiretroviral therapy (ART). Adherence to ART is crucial in optimising health outcomes for PLWH [19-22], and stay-at-home orders potentially impact ART adherence through interlinked individual, social and structural factors. Lockdown may impact ART adherence through structural factors by creating and exacerbating inequalities in access to health care, as well as by impacting food supply chains and generating economic insecurity [23-26]. ART adherence may be affected by the impact of lockdown on social (household) factors such as household functioning, economic and food insecurity; there were furthermore concerns regarding a potential increase in intimate partner violence (IPV) [4, 27-29]. At the individual level, concerns were raised regarding the lockdown's impact on ART adherence as a result of deteriorating mental health [30].

Public health approaches to ART adherence increasingly recognize the role that the immediate environment, and especially the household, play in creating health-enabling environments [31-36]. Masquillier et al. posit that when household members enact behaviours supporting HIV prevention, discussion and disclosure, this 'household HIV competency' has positive effects on ART adherence for PLWH [32]. As the lockdown confined PLWH almost completely within the household setting, specific attention to this context is therefore warranted. HIV is still highly stigmatized, which for example may mean that ART is challenging to adhere to when household members are confined together and medication cannot be taken in secret [30].

To inform policy, it is imperative to understand the impact of lockdown measures on PLWH and ART adherence. First, this study aimed to investigate changes in the 
individual, social and structural factors affecting ART adherence between the pre- and post-level-five lockdown: particularly whether lockdown had impacted mental health, IPV, household functioning, household income, food security and access to health care. Second, the study investigated correlates of ART adherence during the level-five (strictest) lockdown, in order to ascertain whether ART could be impacted by any changes in these factors during the lockdown. Principal hypotheses were: (1) that lockdown would negatively affect mental health, IPV, household functioning and income, food security and access to health care, and (2) that these factors would be associated with respondents' selfassessed ART adherence during the lockdown.

\section{Methods}

\section{Study Design and Ethics}

This study used baseline and follow-up data from participants in the Sinako trial. Sinako ('we can' in isiXhosa) is a cluster-randomised controlled trial on a household ART adherence intervention for PLWH, based in sub-districts of the Cape Metro area, South Africa. Community health workers (CHWs), who deliver the intervention, are linked to health facilities: twelve facilities were therefore randomly selected, resulting in six facilities per trial arm. The trial protocol is detailed elsewhere [31].

A standard ART adherence support service is delivered in both trial arms, with the addition of the Sinako intervention in the intervention arm. The intervention consists of seven hour-long sessions with trial participants and, if the participant agrees, a household member. The household was defined as including all those people who "eat from the same pot' for at least four nights per week over the last month [31]. The intervention sessions cover a variety of topics from adherence planning to developing household HIV competency. By March 2020, patients enrolled in Sinako had received between one and four sessions of the intervention, focusing on HIV knowledge and adherence planning, with the majority having received two sessions: the full intervention had not been received by any patient. This article does not, therefore, specifically focus on the impact of the intervention as it was interrupted at an early stage by the lockdown.

Baseline data was collected between 8th October 2019 and 13th March 2020: fieldworkers interviewed 152 PLWH from 11/12 clinics (56 patients in the control arm, 96 patients in the intervention arm) in English, Afrikaans and isiXhosa before the team halted data collection due to the lockdown. To capture the impact of the lockdown on ART adherence, a follow-up questionnaire was developed. Trained SINAKO field workers attempted to contact all 152 patients by telephone: 69 participants either did not answer the phone or the call could not connect. Between 17th June 2020 and 30th July 2020, 83 patients were re-interviewed. This period fell during South Africa's level three of lockdown restrictions which allowed visitors from one other household, as well as travel for work or education, although other restrictions on movement were still in place. Participants were given prior warning preceding questions on IPV; they were also able to refuse to answer any questions. The fieldworkers were prepared with a list of local organisations and services in the event of being asked for help.

The study was approved by the ethics committee of the University of the Western Cape (BM19/4/6, June 2019) and the ethical committee for the Social Sciences and Humanities of the University of Antwerp (SHW_17_64, September 2018). The City of Cape Town and the Western Cape Department of Health granted permission for all facilities by December 2019. Ethical approval was updated for the follow-up interviews (SHW_17_64 (wijziging), BM19/4/6, August 2020). All participants provided written informed consent at baseline in their chosen language, and oral informed consent during the follow-up telephone questionnaire. Participants were informed that they would receive a shopping voucher following participation in baseline and endline questionnaires.

\section{Measures}

Both baseline and follow-up questionnaires consisted of items to capture socio-economic status (e.g. education level, household income, household expenditure), self-rated ART adherence (0-10 scale), and demographics such as gender and age. An upper-bound poverty line variable was calculated in line with South Africa's 2020 poverty line estimate of 1227 ZAR per person per month (US\$83.60) [37], based on number of people in the household. Household members' age was not accounted for as this information was not available at follow-up. Education was reduced to two categories from five in order to reduce data sparsity whilst retaining thematic relevance.

The baseline survey included one question related to food security ('Does your household ever eat less than it should because there is not enough money for food?') and the follow-up questionnaire included 17 items. Questions on food security were adapted from the South African National Health and Nutrition Examination Survey 2012 [38], including five items on access to food with yes/no responses, and 11 items discussing frequency of e.g. limiting portion sizes, with a 5-point Likert scale response option ranging from 'every day' to 'never'. One item asked whether food insecurity had occured during or after the level-five lockdown. 
Questions on intimate partner violence were adapted from the World Health Organisation's (WHO) multi-country study on women's health and domestic violence [39], which has been widely used in many different contexts and settings. Questions focused on four domains of violence: control, emotional violence, physical violence and sexual violence. The original response options were adapted to ask about (other) household members as well as a current or recent partner: 'No[one]', 'Yes - my current or recent partner in the household', 'Yes-(an)other household member(s)', 'Yes - my current or recent partner outside the household'.

Questions on household stigma were drawn from the 8-item "People Living With HIV Stigma Index" [40]: all eight were used at baseline and three at follow-up, focusing on gossip, blame and insults in the household. Response options included a five-point Likert scale ranging from 'completely agree' to 'completely disagree'. Household functioning was assessed using questions from the validated Brief Family Relationship Scale [41], which uses a fourpoint Likert scale with response options ranging between 'strongly disagree' and 'strongly agree'. Items that were negatively worded were reverse coded so that higher scores represented more positive household functioning. Each question in this scale was analyzed individually given the difficulties of multilevel structural equation modelling with small sample sizes.

Household HIV competency was assessed using 16 questions developed by an author (C. Masquillier) based on qualitative research [32, 33]; five of these questions were used in both the baseline and follow-up questionnaires. Response options included a six-point Likert scale ranging from 'completely disagree' to 'completely agree': one of the five items was reverse coded so that higher scores reflected positive HIV household competency. Questions were once again analysed separately.

Additional questions were developed by the research team for the follow-up questionnaire to ask questions directly related to the lockdown, e.g., 'Would you say that during lockdown [level] 5, you were more depressed?' and 'since I have disclosed my HIV status during lockdown [level] 5, a household member has become more violent towards me'. Response options included five-point Likert scales ranging from 'completely agree' to 'completely disagree'.

\section{Analytical Strategy}

Analysis was undertaken using Stata v.16.0. Descriptive analysis expressed demographic and adherence characteristics as percentages or as mean and median values. Differences between those who participated at baseline and at follow-up were examined using Stata's chi ${ }^{2}$ test/t-test adjusted for clustering within ART clinics [42]. Differences between control and intervention group were also examined using adjusted $\mathrm{chi}^{2} / \mathrm{t}$-tests (Tables 1,2 ). Table VI in the supplementary materials details additional analysis on differences across study arms for those lost to follow-up: there was no statistical evidence of a difference for any of the indicators.

Differences between baseline and follow-up responses were examined using random effects mixed models: time point was included as a fixed effect, while participant ID and ART clinic (health facility) were included as random effects to account for within-person correlation and the unit of randomisation [43]. Correlates of self-reported adherence score at follow-up were analysed using random effects mixed models with ART clinic included as a random effect, and baseline adherence score as a predictor [43].

Following the recommendations of Elff et al. for analyzing multilevel models with few clusters [44], restricted maximum likelihood estimation with a Kenward-Roger correction was used for continuous outcomes to minimize small sample bias [45]. Mixed effects logistic regression with Gauss-Hermite adaptive quadrature was used for binary outcomes. Model validity was assessed using likelihood ratio tests and/or AIC/BIC criterion. Crude and adjusted estimates were calculated: covariates for adjustment were selected based on a priori interest (age, gender, education, household income, intervention group) [46]. Given concerns regarding data sparsity, additional predictors were not added simultaneously to the adjusted model. Sensitivity analysis examined results without adjusting for clustering, as well as using the three-level model for correlates of adherence and the two-level model for changes between baseline and follow-up. Conclusions were not changed based on sensitivity analysis.

The interaction between time point and intervention for ART adherence was examined in order to assess whether this variable should be included in the model. Given that there was weak evidence of an interaction effect between time point and intervention group (the average adherence score increased during the COVID-19 lockdown for participants in almost all of the intervention districts whilst it decreased for those in the control groups), this variable was included in the model: results of the analysis on the impact of the intervention on ART adherence are included in Supplementary Materials (Supplementary material Table III, Table IV, Table V and Supplementary Fig. 1).

\section{Results}

\section{Sample Characteristics}

One clinic had not registered any participants: data from $11 / 12$ clinics was therefore included. Of the $152 \mathrm{PLWH}$ enrolled at baseline, the average age was 31 years (median 
Table 1 Socio-demographic indicators at baseline and follow-up

\begin{tabular}{|c|c|c|c|c|c|c|c|}
\hline & \multicolumn{3}{|l|}{ Baseline } & \multicolumn{3}{|l|}{ Follow-up } & \multirow{3}{*}{$\begin{array}{l}\text { Assoc. } \\
\text { with follow- } \\
\text { up** } \\
x^{2 / t} \& \text { p-value }\end{array}$} \\
\hline & \multicolumn{3}{|l|}{$(\%)$} & \multicolumn{3}{|l|}{$(\%)$} & \\
\hline & $\begin{array}{l}\text { Control } \\
n=56\end{array}$ & $\begin{array}{l}\text { Intervention } \\
\mathrm{n}=96\end{array}$ & $\begin{array}{l}\text { Total } n=152 \\
x^{2 / t} \& \text { p-value** }\end{array}$ & $\begin{array}{l}\text { Control } \\
n=32\end{array}$ & $\begin{array}{l}\text { Intervention } \\
\mathrm{n}=51\end{array}$ & $\begin{array}{l}\text { Total } n=83 \\
\mathrm{x}^{2 / t} \& \mathrm{p}-\text { value }^{* *}\end{array}$ & \\
\hline \multirow[t]{2}{*}{ Age (median) } & & & $0.43, p=0.68$ & & & $0.43 p=0.67$ & \\
\hline & $\begin{array}{l}28.5(\mathrm{IQR} 10.5 \\
\min -\max \\
18-60)\end{array}$ & $\begin{array}{c}29 \text { (IQR 11, } \\
\min -\max \\
19-58)\end{array}$ & $\begin{array}{c}29 \text { (IQR } 11, \\
\min -\max \\
18-60)\end{array}$ & $\begin{array}{c}32(\mathrm{IQR} 10.5 \\
\min -\max \\
18-60)\end{array}$ & $\begin{array}{c}31 \text { (IQR } 13, \\
\min -\max \\
19-58)\end{array}$ & $\begin{array}{c}31 \text { (IQR 11, } \\
\text { min-max } \\
18-60)\end{array}$ & $\begin{array}{l}-1.58 \\
p=0.13\end{array}$ \\
\hline Gender & & & $0.60, p=0.44$ & & & $0.76, p=0.39$ & \\
\hline Female & $39(69.64)$ & $73(76.04)$ & $112(73.68)$ & $21(65.63)$ & $38(74.51)$ & $59(71.08)$ & 0.64 \\
\hline Male & $17(30.36)$ & $23(23.96)$ & $40(26.32)$ & $11(34.38)$ & $13(25.49)$ & $24(28.92)$ & $\mathrm{p}=0.43$ \\
\hline \multirow{3}{*}{$\begin{array}{l}\text { No. of house- } \\
\quad \text { hold members } \\
\text { (median) }\end{array}$} & & & $1.19, p=0.26$ & & & $\begin{array}{c}0.24, p=0.82 \\
p=0.61\end{array}$ & \\
\hline & $\begin{array}{l}3 \text { (IQR 3, min- } \\
\max 0-12)\end{array}$ & $\begin{array}{l}3 \text { (IQR 3, min- } \\
\max 0-8)\end{array}$ & $\begin{array}{l}3 \text { (IQR 3, min- } \\
\max 0-12)\end{array}$ & $\begin{array}{l}2(\mathrm{IQR} 3.5, \\
\min -\max \\
1-12)\end{array}$ & $\begin{array}{l}3 \text { (IQR 3, min- } \\
\max 0-8)\end{array}$ & $\begin{array}{l}3 \text { (IQR 3, min- } \\
\max 0-12)\end{array}$ & -1.04 \\
\hline & & & & & & & $\mathrm{p}=0.31$ \\
\hline Language & & & $0.24, p=0.89$ & & & $0.70, p=0.71$ & \\
\hline Afrikaans & $8(14.29)$ & $10(10.42)$ & $18(11.84)$ & $2(6.25)$ & $8(15.69)$ & $10(12.05)$ & $0.008, p=0.10$ \\
\hline English & $18(32.14)$ & $26(27.08)$ & 44 (28.95) & $11(34.38)$ & $13(25.49)$ & $24(28.92)$ & \\
\hline Xhosa & $30(53.57)$ & $60(62.50)$ & $90(59.21)$ & $19(59.38)$ & $30(58.82)$ & $49(59.04)$ & \\
\hline Education & & & $1.05, p=0.90$ & & & $0.68, p=0.88$ & $8.46, p=0.08$ \\
\hline None & 0 & $1(1.04)$ & $1(0.66)$ & 0 & 0 & 0 & \\
\hline Primary & $14(25)$ & $15(15.63)$ & $29(19.08)$ & $8(25)$ & $10(19.61)$ & $18(21.69)$ & $\begin{array}{c}\text { Binary: } 4.74 \\
p=0.03\end{array}$ \\
\hline Secondary & $27(48.21)$ & $46(47.92)$ & 73 (48.03) & $14(43.75)$ & $18(35.29)$ & $32(38.55)$ & \\
\hline Matric & $14(25)$ & $31(32.29)$ & $45(29.61)$ & $9(28.13)$ & $22(43.14)$ & $31(37.35)$ & \\
\hline Diploma & $1(1.79)$ & $3(3.13)$ & $4(2.63)$ & $1(3.13)$ & $1(1.96)$ & $2(2.41)$ & \\
\hline $\begin{array}{r}\text { Well-being } \\
\text { (median) }\end{array}$ & & & $-1.59, p=0.15$ & & & $-0.80, p=0.45$ & $1.17, p=0.26$ \\
\hline Scale of $0-100$ & $\begin{array}{c}90(\mathrm{IQR} 30 \\
\min -\max \\
5-100)\end{array}$ & $\begin{array}{l}99.5 \text { (IQR 20, } \\
\text { min-max 50- } \\
100)\end{array}$ & $\begin{array}{c}97 \text { (IQR 20, } \\
\min -\max \\
5-100)\end{array}$ & $\begin{array}{c}96.5 \text { (IQR 20, } \\
\min -\max \\
30-100)\end{array}$ & $\begin{array}{c}100(\text { IQR } 10 \\
\min -\max \\
60-100)\end{array}$ & $\begin{array}{c}100(\text { IQR } 15 \\
\min -\max \\
30-100)\end{array}$ & \\
\hline \multirow{2}{*}{$\begin{array}{l}\text { Household } \\
\text { income (ZAR } \\
\text { per month) }\end{array}$} & & & $(n=126)$ & & & $(\mathrm{n}=76)$ & \\
\hline & & & $1.21, p=0.55$ & & & $2.19, p=0.23$ & $1.60, p=0.45$ \\
\hline $0-2000$ & $11(22.92)$ & $23(29.49)$ & $34(26.98)$ & $15(48.39)$ & $26(57.78)$ & $41(53.95)$ & \\
\hline $2000-5000$ & $20(41.67)$ & 34 (43.59) & $54(42.86)$ & $13(41.94)$ & $11(24.44)$ & $24(31.58)$ & \\
\hline $5000-50 \mathrm{k}$ & $17(35.42)$ & $21(26.92)$ & $38(30.16)$ & $3(9.68)$ & $8(17.78)$ & $11(14.47)$ & \\
\hline
\end{tabular}

** $\mathrm{Chi}^{2} / \mathrm{t}$-test adjusted for clustering within ART clinic

28.5 years), with 112 women (74\%) and 40 men (26\%) (Table 1). The follow-up questionnaire was completed by 83 participants, of whom 59 were women $(71 \%)$ and 24 were men (29\%), with an average age of 33 years (median 31). There was no evidence of an association between socio-demographic variables and whether a respondent participated in the follow-up questionnaire, except for education level (Tables 1, 2). At baseline, 103 participants had either no education, primary education or some secondary education (68\%), and 49 had obtained either their secondary matriculation, a degree or a diploma (32\%): for those who completed the follow-up questionnaire, these categories comprised 50 participants $(60 \%)$ and 33 participants (40\%) respectively. The odds of participating in the followup for those with higher levels of education were twice as high compared to those with lower levels of education (OR $2.22, \mathrm{z}=2.13, \mathrm{p}=0.03)$. 
Table 2 Socio-demographic indicators at baseline and follow-up

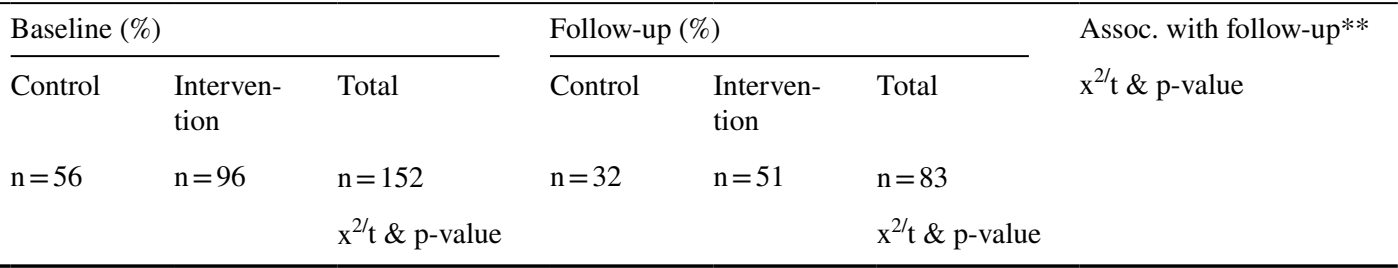

\begin{tabular}{|c|c|c|c|c|c|c|c|}
\hline ART clinic & & & $(*)$ & & & & \\
\hline 1 & 0 & $17(17.71)$ & 17 & 0 & $10(19.61)$ & 10 & \\
\hline 2 & $23(41.07)$ & 0 & 23 & $15(46.88)$ & 0 & 15 & \\
\hline 3 & $6(10.71)$ & 0 & 6 & $1(3.13)$ & 0 & 1 & \\
\hline 4 & 0 & $28(29.17)$ & 28 & 0 & $12(23.53)$ & 12 & 10.03 \\
\hline 5 & 0 & $11(11.46)$ & 11 & 0 & $6(11.76)$ & 6 & $\mathrm{p}=$ \\
\hline 6 & $8(14.29)$ & 0 & 8 & $3(9.38)$ & 0 & 3 & $0.44 * * *$ \\
\hline 7 & $10(17.86)$ & 0 & 10 & $8(25)$ & 0 & 8 & \\
\hline 8 & 0 & $27(28.13)$ & 27 & 0 & $16(31.37)$ & 16 & \\
\hline 9 & 0 & $4(4.17)$ & 4 & 0 & $2(3.92)$ & 2 & \\
\hline 10 & 0 & $9(9.38)$ & 9 & 0 & $5(9.80)$ & 5 & \\
\hline 11 & $9(16.07)$ & 0 & 9 & $5(15.63)$ & 0 & 5 & \\
\hline Water & & & $10.35, p=0.07$ & & & $3.85, p=0.80$ & \\
\hline Pipe, meter & $19(33.93)$ & 43 (44.79) & $62(40.79)$ & $8(25)$ & $24(47.06)$ & $32(38.55)$ & \\
\hline Pipe, prepay & $4(7.14)$ & $2(2.08)$ & $6(3.95)$ & 0 & $1(1.96)$ & $1(1.20)$ & $5.03, p=0.41$ \\
\hline Piped, yard & $11(19.64)$ & $28(29.17)$ & 39 (25.66) & $10(31.25)$ & $13(25.49)$ & $23(27.71)$ & \\
\hline Public, free & $20(35.71)$ & $20(20.83)$ & $40(26.32)$ & $12(37.50)$ & $10(19.61)$ & $22(26.51)$ & \\
\hline Public, paid & $2(3.57)$ & 0 & $2(1.32)$ & 0 & $1(1.96)$ & $1(1.20)$ & \\
\hline Rainwater & 0 & 0 & 0 & 0 & 1 & $1(1.20)$ & \\
\hline Bottled & 0 & 0 & 0 & 0 & 0 & 0 & \\
\hline Water tanks & - & - & 0 & $2(6.25)$ & 0 & $2(2.41)$ & \\
\hline Other & 0 & 0 & $3(1.97)$ & 0 & $1(1.96)$ & $1(1.20)$ & \\
\hline Electricity & & & $4.07, p=0.54$ & & & $4.60, p=0.47$ & $4.05, p=0.54$ \\
\hline Home meter & $11(19.64)$ & $23(23.96)$ & $34(22.37)$ & $5(15.63)$ & $11(21.57)$ & $16(19.28)$ & \\
\hline Home prepay & $28(50)$ & $54(56.25)$ & $82(53.95)$ & $16(50)$ & $32(62.75)$ & $48(57.83)$ & \\
\hline Other (free) & $8(14.29)$ & $14(14.58$ & $22(14.47)$ & $6(18.75)$ & $5(9.80)$ & $11(13.25)$ & \\
\hline Other (pay) & $3(5.36)$ & 0 & $3(1.97)$ & $3(9.38)$ & 0 & $3(3.61)$ & \\
\hline Illegal conn & $2(3.57)$ & $5(5.21)$ & $7(4.61)$ & $1(3.13)$ & $3(5.88)$ & $4(4.82)$ & \\
\hline No source & $4(7.14)$ & 0 & $4(2.63)$ & $1(3.13)$ & 0 & $1(1.20)$ & \\
\hline \multicolumn{8}{|c|}{ HH eats less than should } \\
\hline No & & & $0.07, p=0.80$ & - & - & - & $3.04, p=0.08$ \\
\hline \multirow[t]{2}{*}{ Yes } & $21(37.50)$ & $38(39.58)$ & $59(38.82)$ & & & & \\
\hline & $35(62.50)$ & $58(60.42)$ & $93(61.18)$ & & & & \\
\hline
\end{tabular}

*ART clinic was the unit of randomisation

** $\mathrm{Chi}^{2} / \mathrm{t}$-test adjusted for clustering within ART clinic

$* * * \mathrm{Chi}^{2}$ test

\section{Differences Between Baseline and Follow-Up}

This study first examined whether there were changes in the individual, household and structural factors that may affect
ART adherence between baseline and follow-up. There was no evidence of a difference in average adherence scores between baseline and follow-up, holding other variables constant $(0.049, z=0.20, p=0.84$, Table 3$)$. 
Table 3 Individual and household factors: differences between baseline and follow-up

\begin{tabular}{|c|c|c|c|c|c|}
\hline \multicolumn{2}{|l|}{ Variable } & \multirow[t]{2}{*}{$\begin{array}{l}\text { Baseline } \\
(\mathrm{n}=152)(\%)\end{array}$} & \multirow[t]{2}{*}{$\begin{array}{l}\text { Follow-Up }(\mathrm{n}=83) \\
(\%)\end{array}$} & \multirow{2}{*}{$\begin{array}{l}\text { Odds Ratio / Esti- } \\
\text { mate*, baseline vs } \\
\text { follow-up } \\
\text { z \& p-value }\end{array}$} & \multirow{2}{*}{$\begin{array}{l}\text { Adjusted Odds Ratio / } \\
\text { Estimate** } \\
\text { z \& p-value }\end{array}$} \\
\hline \multicolumn{2}{|c|}{ Well-being, income and poverty line } & & & & \\
\hline Adherence & Self-rated scale $0-10$ & $\begin{array}{l}\text { mean } 9.31 \\
(\text { std dev } 1.52)\end{array}$ & $\begin{array}{l}\text { mean } 9.24 \\
(\text { std dev } 1.64)\end{array}$ & $\begin{array}{c}\text { Coef:- } 0.04,(\mathrm{SE} \\
0.21),-0.21 \\
\mathrm{p}=0.84\end{array}$ & $\begin{array}{l}\text { Coef: } 0.049,(\text { SE 0.24), } \\
0.20, p=0.84\end{array}$ \\
\hline Well-being & Scale $0-100$ & $\begin{array}{l}\text { mean } 85.59, \text { med. } 97 \\
\text { (IQR 20, min-max } \\
5-100)\end{array}$ & $\begin{array}{l}\text { mean } 91.81 \text { med.100 } \\
\text { (IQR 15, min-max } \\
\text { 30-100) }\end{array}$ & $\begin{array}{l}\text { Coef: } 6.94,(\text { SE } 2.06) \\
\text { 3.37, } p=0.001\end{array}$ & $\begin{array}{l}\text { Coef: } 9.50,(\text { SE } 2.29) \\
4.16, p<0.001\end{array}$ \\
\hline \multirow{4}{*}{$\begin{array}{l}\text { Household Income } \\
\text { (ZAR per month) }\end{array}$} & 0-2000 & $34(26.98)$ & $41(53.95)$ & \multirow{4}{*}{$\begin{array}{l}\text { OR 0.21, (SE 0.08), } \\
\quad-3.92, \mathrm{p}<0.001\end{array}$} & \multirow{4}{*}{$\begin{array}{l}\text { aOR 0.21, (SE 0.08), } \\
-4.01, \mathrm{p}<0.001\end{array}$} \\
\hline & $2000-5000$ & $54(42.86)$ & $24(31.58)$ & & \\
\hline & $5000-50 \mathrm{k}$ & $38(30.16)$ & $11(14.47)$ & & \\
\hline & Total & $126(100)$ & $76(100)$ & & \\
\hline \multirow{3}{*}{$\begin{array}{l}\text { Upper bound poverty } \\
\text { line }\end{array}$} & Above line & $45(35.71)$ & $16(21.05)$ & \multirow{3}{*}{$\begin{array}{l}\text { OR 2.40, (SE 0.96), } \\
2.17, \mathrm{p}=0.03\end{array}$} & \multirow{3}{*}{$\begin{array}{l}\text { aOR 2.41, (SE 0.97), } \\
2.19, \mathrm{p}=0.03\end{array}$} \\
\hline & Below line & $81(64.29)$ & $60(78.95)$ & & \\
\hline & Total & $126(100)$ & $76(100)$ & & \\
\hline \multicolumn{4}{|c|}{ Household stigma: because of my HIV status... } & \multicolumn{2}{|l|}{ (Binary variable) } \\
\hline \multirow{5}{*}{$\begin{array}{l}\text { I am being gossiped } \\
\text { about in my house- } \\
\text { hold }\end{array}$} & Comp. disagree & $26(18.44)$ & $34(40.96)$ & \multirow{5}{*}{$\begin{array}{l}\text { OR: 0.54, (SE 0.29), } \\
\quad-1.15, \mathrm{p}=0.25\end{array}$} & \multirow{5}{*}{$\begin{array}{l}\text { aOR: } 0.43,(\operatorname{SE~} 0.25) \\
\quad-1.46, p=0.15\end{array}$} \\
\hline & Disagree & $100(70.92)$ & $44(53.01)$ & & \\
\hline & Neither a/d & $7(4.96)$ & $1(1.20)$ & & \\
\hline & Agree & $5(3.55)$ & $3(3.61)$ & & \\
\hline & Comp. agree & $3(2.13)$ & $1(1.20)$ & & \\
\hline \multirow{5}{*}{$\begin{array}{l}\text { I feel blamed by my } \\
\text { household members }\end{array}$} & Comp. disagree & $27(19.15)$ & $30(36.14)$ & \multirow{5}{*}{$\begin{array}{l}\text { OR: } 0.25,(\text { SE } 0.16) \\
\quad-2.15, p=0.03\end{array}$} & \multirow{5}{*}{$\begin{array}{l}\text { aOR: } 0.15,(\text { SE 0.12) } \\
\quad-2.33, p=0.02\end{array}$} \\
\hline & Disagree & $96(68.09)$ & $50(60.24)$ & & \\
\hline & Neither a/d & $6(4.26)$ & $1(1.20)$ & & \\
\hline & Agree & $11(7.80)$ & $1(1.20)$ & & \\
\hline & Comp. agree & $1(0.71)$ & $1(1.20)$ & & \\
\hline \multirow{5}{*}{$\begin{array}{l}\text { I am verbally insulted } \\
\text { and harassed by my } \\
\text { household members }\end{array}$} & Comp. disagree & $40(28.37)$ & $39(46.99)$ & \multirow{5}{*}{$\begin{array}{l}\text { OR: } 0.61,(\text { SE } 0.42) \\
\quad-0.71, p=0.48\end{array}$} & \multirow{5}{*}{$\begin{array}{l}\text { aOR: } 0.57,(\text { SE 0.44) } \\
\quad-0.73, p=0.47\end{array}$} \\
\hline & Disagree & $93(65.96)$ & $41(49.40)$ & & \\
\hline & Neither a/d & $6(4.26)$ & $1(1.20)$ & & \\
\hline & Agree & $2(1.42)$ & $1(1.20)$ & & \\
\hline & Comp. agree & $0(0)$ & $1(1.20)$ & & \\
\hline Household HIV compe & tency: (with) my househ & old members... & & & \\
\hline I share HIV worries & 0 Agree & $112(73.68)$ & $70(84.34)$ & OR 0.42, (SE 0.19)), & aOR: 0.43, (SE 0.24), \\
\hline & 1 Disagree & $40(26.32)$ & $13(15.66)$ & $-1.91, p=0.06$ & $-1.53, p=0.13$ \\
\hline Help remember medi- & 0 Agree & $115(75.66)$ & $70(84.34)$ & OR 0.51, (SE 0.22), & aOR: 0.49, (SE 0.22), \\
\hline cation & 1 Disagree & 37 (24.34) & $13(15.66)$ & & $-1.54, p=0.12$ \\
\hline Give emotional sup- & 0 Agree & $119(78.29)$ & $72(86.75)$ & OR 0.48, (SE 0.25), & aOR: 0.44, (SE 0.29), \\
\hline port & 1 Disagree & $33(21.71)$ & $11(13.25)$ & $-1.41, p=0.16$ & $-1.27, \mathrm{p}=0.21$ \\
\hline I can talk openly & 0 Agree & $105(69.08)$ & $61(73.49)$ & OR 0.68, (SE 0.26), & aOR: 0.91, (SE 0.39), \\
\hline & 1 Disagree & $47(30.92)$ & $22(26.51)$ & $-1.00, p=0.32$ & $-0.21, p=0.83$ \\
\hline I can solve difficulties & 0 Agree & $121(79.61)$ & $65(78.31)$ & OR 1.08, (SE 0.36), & aOR: 1.50, (SE 0.57), \\
\hline & 1 Disagree & $31(20.39)$ & $18(21.69)$ & $0.23, p=0.82$ & $1.07, \mathrm{p}=0.28$ \\
\hline
\end{tabular}

*Three-level mixed effects model, time as a fixed effect and ART clinic and participant as random effects

** Model adjusted for age, gender, education, household income (except when included as a dependent variable), intervention group

\section{Structural Factors: Health Care Access and Food Insecurity}

A minority of participants reported issues with accessing health care to support their ART adherence while a majority reported feeling worried about access: at follow-up, 13 $(15.66 \%)$ respondents stated that because of the lockdown, they had missed an appointment at the clinic for their HIV 
treatment or care (Supplementary material, Table II). 47 (56.63\%) respondents worried that they would run out of their HIV medication, and 11 (13.25\%) stated that they had in fact run out of medication. The majority of respondents $(58,69.88 \%)$ stated that they missed the CHW's support.

The majority of participants also reported issues obtaining food during the lockdown (Supplementary material, Table I). 50 (60\%) reported having problems accessing food, 43 of whom stated this was due to economic difficulties. 30 respondents $(60 \%)$ stated that they had problems accessing food because the shops were difficult to get to during the lockdown.

\section{Social (Household) Factors: Household Food Insecurity, Economic Insecurity, Stigma, Violence, and Household Functioning}

$53(64 \%)$ respondents reported eating less than normal. Almost 22\% [18] often restricted the consumption of adults in order for children to eat, and 40\% (33 respondents) limited portion sizes at mealtimes. The odds of reducing portion sizes were lower for those with a household income of over $5,000 \mathrm{ZAR}$ a month as compared to lower categories of household income (aOR: 0.37, $\mathrm{z}=-2.73, \mathrm{p}=0.006$ ).

The analysis indicated that the economic situation of households had deteriorated during the lockdown. Between baseline and follow-up, the odds of being in the top household income category decreased (aOR $0.21, \mathrm{z}=-4.01$, $\mathrm{p}<0.001)$ and participants had 2.41 times greater odds of being below the poverty line $(\mathrm{z}=2.19, \mathrm{p}=0.03)$ after adjusting for socio-demographic variables (Table 3 ).

The analysis indicated some improvements in indicators relating to household environments after the period of the lockdown. There was a decrease in the odds of experiencing household HIV stigma between baseline and follow-up for those feeling blamed by their household members because of their HIV status (aOR: $0.15,-2.33, \mathrm{p}=0.02$ ) (Table 3 ). There was similarly evidence of a decrease in reporting any instance of violence (emotional, physical, sexual) between baseline and follow-up (aOR: 0.08, $\mathrm{z}=-2.2, \mathrm{p}=0.024$ ) (Table 4). All participants who reported violence at followup had stated at baseline that the perpetrator was a current

Table 4 Violence at baseline and follow-up

\begin{tabular}{|c|c|c|c|c|c|c|c|}
\hline & Response categories & Baseline (\%) & Follow-up (\%) & \multicolumn{2}{|c|}{$\begin{array}{l}\text { Odds ratio/coefficient*, } \\
\text { baseline vs follow-up } \\
\text { z \& p-value }\end{array}$} & \multicolumn{2}{|c|}{$\begin{array}{l}\text { Adjusted odds ratio/ } \\
\text { coefficient* } \\
\text { z \& p-value }\end{array}$} \\
\hline $\begin{array}{l}\text { Insulted you/ made you } \\
\text { feel bad }\end{array}$ & $\begin{array}{l}\text { No } \\
\text { Yes }\end{array}$ & $\begin{array}{l}132(86.84) \\
20(13.16)\end{array}$ & $\begin{array}{l}79(95.18) \\
4(4.82)\end{array}$ & \multicolumn{2}{|c|}{$\begin{array}{l}\text { OR: } 0.31,(\text { SE } 0.19) \\
\quad-1.90, p=0.06\end{array}$} & \multicolumn{2}{|c|}{$\begin{array}{l}\text { aOR: } 0.27,(\text { SE } 0.21) \\
\quad-1.73, p=0.08\end{array}$} \\
\hline $\begin{array}{l}\text { Scared or intimidated } \\
\text { you }\end{array}$ & $\begin{array}{l}\text { No } \\
\text { Yes }\end{array}$ & $\begin{array}{l}143(94.08) \\
9(5.92)\end{array}$ & $\begin{array}{l}80(96.39) \\
3(3.61)\end{array}$ & \multicolumn{2}{|c|}{$\begin{array}{l}\text { OR: } 0.07,(\text { SE } 0.33) \\
\quad-1.09, p=0.27\end{array}$} & \multicolumn{2}{|c|}{$\begin{array}{l}\text { aOR: } 0.50,(\text { SE } 0.63) \\
\quad-0.56, p=0.58\end{array}$} \\
\hline $\begin{array}{l}\text { Slapped or threw some- } \\
\text { thing at you }\end{array}$ & $\begin{array}{l}\text { No } \\
\text { Yes }\end{array}$ & $\begin{array}{l}137(90.13) \\
15(9.87)\end{array}$ & $\begin{array}{l}83(100.00) \\
0(0.00)\end{array}$ & \multicolumn{2}{|l|}{-} & \multicolumn{2}{|l|}{-} \\
\hline Forced you to have sex & $\begin{array}{l}\text { No } \\
\text { Yes }\end{array}$ & $\begin{array}{l}148(97.37) \\
4(2.63)\end{array}$ & $\begin{array}{l}83(100.00) \\
0(0.00)\end{array}$ & \multicolumn{2}{|l|}{-} & \multicolumn{2}{|l|}{-} \\
\hline $\begin{array}{l}\text { Had sex as afraid if } \\
\text { refused }\end{array}$ & $\begin{array}{l}\text { No } \\
\text { Yes }\end{array}$ & $\begin{array}{l}148(97.37) \\
4(2.63)\end{array}$ & $\begin{array}{l}82(98.80) \\
1(1.21)\end{array}$ & \multicolumn{2}{|c|}{$\begin{array}{l}\text { OR: } 0.45,(\text { SE } 0.51) \\
\quad-0.71, p=0.48\end{array}$} & \multicolumn{2}{|c|}{$\begin{array}{l}\text { aOR: } 0.64(\mathrm{SE} 0.82) \\
\quad-0.35, \mathrm{p}=0.73\end{array}$} \\
\hline Any instance of violence & $\begin{array}{l}\text { None } \\
\text { Violence }\end{array}$ & $\begin{array}{l}118(77.63) \\
34(22.37)\end{array}$ & $\begin{array}{l}77(92.77) \\
6(7.23)\end{array}$ & \multicolumn{2}{|c|}{$\begin{array}{l}\text { OR: } 0.14,(\text { SE } 0.10) \\
\quad-2.70, p=0.007\end{array}$} & \multicolumn{2}{|c|}{$\begin{array}{l}\text { aOR: } 0.08(\text { SE 0.09), } \\
\quad-2.2, p=0.024\end{array}$} \\
\hline $\begin{array}{l}\text { Violence: During } \\
\text { lockdown, were you } \\
{[\ldots] \text { (by a household }} \\
\text { member): }\end{array}$ & Response categories & Frequencies (\%) & & $\begin{array}{l}\text { Violence: During lock- } \\
\text { down, were you }[\ldots] \text { : }\end{array}$ & Response catego & pries & Frequencies $(\%)$ \\
\hline $\begin{array}{l}\text { Insulted more or less } \\
\text { than before lock- } \\
\text { down? }\end{array}$ & $\begin{array}{l}\text { Less } \\
\text { Same } \\
\text { More }\end{array}$ & $\begin{array}{l}0(0.00) \\
1(25.00) \\
3(75.00)\end{array}$ & & $\begin{array}{l}\text { Had sex that you didn't } \\
\text { want more or less } \\
\text { than before? }\end{array}$ & $\begin{array}{l}\text { Less than before } \\
\text { About the same } \\
\text { More than befor }\end{array}$ & & $\begin{array}{l}0(0.00) \\
0(0.00) \\
1(100.00)\end{array}$ \\
\hline $\begin{array}{l}\text { Scared or intimidated } \\
\text { more or less than } \\
\text { before? }\end{array}$ & $\begin{array}{l}\text { Less } \\
\text { Same } \\
\text { More }\end{array}$ & $\begin{array}{l}1(33.33) \\
1(33.33) \\
1(33.33)\end{array}$ & & $\begin{array}{l}\text { Since I disclosed HIV } \\
\text { during lockdown, } \\
\text { a hhm** is more } \\
\text { violent }\end{array}$ & $\begin{array}{l}\text { Strongly disagre } \\
\text { Disagree } \\
\text { Agree } \\
\text { Strongly agree } \\
\text { Have not disclos }\end{array}$ & & $\begin{array}{l}41(49.40) \\
35(42.17) \\
1(1.20) \\
1(1.20) \\
5(6.02)\end{array}$ \\
\hline
\end{tabular}

*Three-level mixed effects model, time as a fixed effect and ART clinic and participant as random effects

**Hhm refers to household member 
Table 5 Household functioning at baseline and follow-up: "in our household..."

\begin{tabular}{|c|c|c|c|c|c|}
\hline Variable & & $\begin{array}{l}\text { Baseline } \\
(\mathrm{n}=152)(\%)\end{array}$ & $\begin{array}{l}\text { Follow-up } \\
(\mathrm{n}=83) \\
(\%)\end{array}$ & Odds ratio, z \& p-value, $95 \%$ CI** & Adjusted OR, z \& p-value $* * *$ \\
\hline We spend time together at home & $\begin{array}{l}\text { Agree } \\
\text { Disagree }\end{array}$ & $\begin{array}{l}120(78.95) \\
32(21.05)\end{array}$ & $\begin{array}{l}77(92.77) \\
6(7.23)\end{array}$ & $\begin{array}{l}\text { OR: } 0.21,-2.59, p=0.010 \\
(95 \% \text { CI } 0.06-0.68)\end{array}$ & $\begin{array}{l}\text { aOR: } 0.32,-1.92, p=0.06 \\
(95 \% \text { CI } 0.10-1.03)\end{array}$ \\
\hline We work hard at what we do & $\begin{array}{l}\text { Agree } \\
\text { Disagree }\end{array}$ & $\begin{array}{l}128(84.21) \\
24(15.79)\end{array}$ & $\begin{array}{l}79(95.18) \\
4(4.82)\end{array}$ & $\begin{array}{l}\text { OR } 0.25,-2.28, p=0.023 \\
(95 \% \text { CI } 0.08-0.82)\end{array}$ & $\begin{array}{l}\text { aOR: } 0.42,-1.43, p=0.15 \\
(95 \% \text { CI } 0.12-1.38)\end{array}$ \\
\hline There is a feeling of togetherness & $\begin{array}{l}\text { Agree } \\
\text { Disagree }\end{array}$ & $\begin{array}{l}123(80.92) \\
29(19.08)\end{array}$ & $\begin{array}{l}79(95.18) \\
4(4.82)\end{array}$ & $\begin{array}{l}\text { OR: } 0.20,-2.64, p=0.008 \\
(95 \% \text { CI } 0.06-0.66)\end{array}$ & $\begin{array}{l}\text { aOR: } 0.29,-2.10, p=0.04 \\
(95 \% \text { CI } 0.09-0.92)\end{array}$ \\
\hline We get along well w. each other & $\begin{array}{l}\text { Agree } \\
\text { Disagree }\end{array}$ & $\begin{array}{l}127(83.55) \\
25(16.45)\end{array}$ & $\begin{array}{l}80(96.39) \\
3(3.61)\end{array}$ & $\begin{array}{l}\text { OR } 0.009,-2.10, p=0.04 \\
(95 \% \text { CI } 0.002-0.73)\end{array}$ & $\begin{array}{l}\text { aOR: } 0.003,-1.46, p=0.14 \\
(95 \% \text { CI } 0.0002-7.35)\end{array}$ \\
\hline We do things for each other & $\begin{array}{l}\text { Agree } \\
\text { Disagree }\end{array}$ & $\begin{array}{l}121(79.61) \\
31(20.39)\end{array}$ & $\begin{array}{l}69(83.13) \\
14(16.87)\end{array}$ & $\begin{array}{l}\text { OR } 0.79,-0.65, p=0.51 \\
(95 \% \text { CI } 0.40-1.59)\end{array}$ & $\begin{array}{l}\text { aOR: } 0.96,-0.11, p=0.92 \\
(95 \% \text { CI } 0.43-2.11)\end{array}$ \\
\hline We work out our problems & $\begin{array}{l}\text { Agree } \\
\text { Disagree }\end{array}$ & $\begin{array}{l}135(88.82) \\
17(11.18)\end{array}$ & $\begin{array}{l}80(96.39) \\
3(3.61)\end{array}$ & $\begin{array}{l}\text { OR } 0.14,-1.89, p=0.06 \\
(95 \% \text { CI } 0.02-1.08)\end{array}$ & $\begin{array}{l}\text { aOR: } 0.19,-1.51, \mathrm{p}=0.13 \\
(95 \% \text { CI } 0.02-1.63)\end{array}$ \\
\hline We can talk openly & $\begin{array}{l}\text { Agree } \\
\text { Disagree }\end{array}$ & $\begin{array}{l}114(75.00) \\
38(25.00)\end{array}$ & $\begin{array}{l}70(84.34) \\
13(15.66)\end{array}$ & $\begin{array}{l}\text { OR } 0.39,-1.92, p=0.06 \\
(95 \% \text { CI } 0.15-1.02)\end{array}$ & $\begin{array}{l}\text { aOR: } 0.53,-1.25, \mathrm{p}=0.21 \\
(95 \% \text { CI } 0.20-1.43)\end{array}$ \\
\hline $\begin{array}{l}\text { We tell each other personal prob- } \\
\text { lems }\end{array}$ & $\begin{array}{l}\text { Agree } \\
\text { Disagree }\end{array}$ & $\begin{array}{l}119(78.29) \\
33(21.71)\end{array}$ & $\begin{array}{l}73(87.95) \\
10(12.05)\end{array}$ & $\begin{array}{l}\text { OR } 0.43,-1.88, p=0.06 \\
(95 \% \text { CI } 0.18-1.04)\end{array}$ & $\begin{array}{l}\text { aOR: } 0.59,-1.18, p=0.24 \\
(95 \% \text { CI } 0.25-1.41)\end{array}$ \\
\hline We begin discussions easily & $\begin{array}{l}\text { Agree } \\
\text { Disagree }\end{array}$ & $\begin{array}{l}121(79.61) \\
31(20.39)\end{array}$ & $\begin{array}{l}74(89.16) \\
9(10.84)\end{array}$ & $\begin{array}{l}\text { OR } 0.39,-1.95, p=0.05 \\
(95 \% \text { CI } 0.15-1.01)\end{array}$ & $\begin{array}{l}\text { aOR: } 0.54,-1.18, p=0.24 \\
(95 \% \text { CI } 0.19-1.50)\end{array}$ \\
\hline We are careful about what we say & $\begin{array}{l}\text { Agree } \\
\text { Disagree }\end{array}$ & $\begin{array}{l}121(79.61) \\
31(20.39)\end{array}$ & $\begin{array}{l}75(90.36) \\
8(9.64)\end{array}$ & $\begin{array}{l}\text { OR } 0.42,-2.07, p=0.04 \\
(95 \% \text { CI } 0.18-0.95)\end{array}$ & $\begin{array}{l}\text { aOR: } 0.50,-1.49, \mathrm{p}=0.14 \\
(95 \% \text { CI } 0.20-1.24)\end{array}$ \\
\hline We argue a lot & $\begin{array}{l}\text { Agree } \\
\text { Disagree }\end{array}$ & $\begin{array}{l}63(41.45) \\
89(58.55)\end{array}$ & $\begin{array}{l}29(34.94) \\
54(65.06)\end{array}$ & $\begin{array}{l}\text { OR } 1.38,1.05, p=0.29 \\
(95 \% \text { CI } 0.76-2.53)\end{array}$ & $\begin{array}{l}\text { aOR: } 1.54,1.26, p=0.29 \\
(95 \% \text { CI } 0.79-3.01)\end{array}$ \\
\hline We are mad at each other a lot & $\begin{array}{l}\text { Agree } \\
\text { Disagree }\end{array}$ & $\begin{array}{l}42(27.63) \\
110(72.37)\end{array}$ & $\begin{array}{l}19(22.89) \\
64(77.11)\end{array}$ & $\begin{array}{l}\text { OR } 1.24,0.52, p=0.61 \\
(95 \% \text { CI } 0.55-2.80)\end{array}$ & $\begin{array}{l}\text { aOR: } 1.42,0.76, p=0.45 \\
(95 \% \text { CI } 0.58-3.48)\end{array}$ \\
\hline We lose our tempers a lot & $\begin{array}{l}\text { Agree } \\
\text { Disagree }\end{array}$ & $\begin{array}{l}40(26.32) \\
112(73.68)\end{array}$ & $\begin{array}{l}14(16.87) \\
69(83.13)\end{array}$ & $\begin{array}{l}\text { OR } 1.85,1.56, p=0.12 \\
(95 \% \text { CI } 0.86-3.99)\end{array}$ & $\begin{array}{l}\text { aOR: } 2.53,1.95, p=0.05 \\
(95 \% \text { CI } 1.00-6.42)\end{array}$ \\
\hline We often put each other down & $\begin{array}{l}\text { Agree } \\
\text { Disagree }\end{array}$ & $\begin{array}{l}29(19.08) \\
123(80.92)\end{array}$ & $\begin{array}{l}11(13.25) \\
72(86.75)\end{array}$ & $\begin{array}{l}\text { OR } 2.14,1.31, p=0.19 \\
(95 \% \text { CI } 0.69-6.69)\end{array}$ & $\begin{array}{l}\text { aOR: } 1.95,1.06, p=0.29 \\
(95 \% \text { CI } 0.57-6.71)\end{array}$ \\
\hline My hhms* are sometimes violent & $\begin{array}{l}\text { Agree } \\
\text { Disagree }\end{array}$ & $\begin{array}{l}36(23.68) \\
116(76.32)\end{array}$ & $\begin{array}{l}12(14.46) \\
71(85.54)\end{array}$ & $\begin{array}{l}\text { OR } 2.09,1.75, p=0.08 \\
(95 \% \text { CI } 0.92-4.79)\end{array}$ & $\begin{array}{l}\text { aOR: } 1.69,1.17, p=0.24 \\
(95 \% \text { CI } 0.70-4.11)\end{array}$ \\
\hline We raise voices when mad & $\begin{array}{l}\text { Agree } \\
\text { Disagree }\end{array}$ & $\begin{array}{l}59(38.82) \\
93(61.18)\end{array}$ & $\begin{array}{l}25(30.12) \\
58(69.88)\end{array}$ & $\begin{array}{l}\text { OR } 1.66,1.47, p=0.14 \\
(95 \% \text { CI } 0.85-3.27)\end{array}$ & $\begin{array}{l}\text { aOR: } 1.68,1.37, p=0.17 \\
(95 \% \text { CI } 0.80-3.53)\end{array}$ \\
\hline $\begin{array}{l}\text { Their support for HIV treatment } \\
\text { changed }\end{array}$ & $\begin{array}{l}\text { Worse } \\
\text { The same } \\
\text { Improved }\end{array}$ & $\begin{array}{l}- \\
- \\
-\end{array}$ & $\begin{array}{l}4(4.82) \\
73(87.95) \\
6(7.23)\end{array}$ & - & - \\
\hline Lockdown brought us closer & $\begin{array}{l}\text { Agree } \\
\text { Disagree }\end{array}$ & - & $\begin{array}{l}81(97.59) \\
2(2.41)\end{array}$ & - & - \\
\hline Lockdown caused more tension & $\begin{array}{l}\text { Agree } \\
\text { Disagree }\end{array}$ & - & $\begin{array}{l}17(20.48) \\
66(79.52)\end{array}$ & - & - \\
\hline
\end{tabular}

*Household members (hhms)

**Three-level mixed effects model, time as a fixed effect and ART clinic and participant as random effects

***Adjusted for age, gender, education, household income, intervention group

partner or household member living in the household-not a partner external to the household. Two respondents furthermore stated that a household member had become more violent since they had disclosed their HIV status during the lockdown (Table 4). At follow-up there was evidence of a decrease in the odds of not having a feeling of togetherness in the household (aOR: $0.29 \mathrm{z}=-2.10, \mathrm{p}=0.04$ ) (Table 5). 


\section{Individual Factors: Self-reported Well-Being and Depression}

There appeared to be improvements in well-being after the period of the lockdown: there was strong evidence of an increase in the self-reported well-being score between baseline and follow-up $(9.50, \mathrm{z}=4.16, \mathrm{p}<0.001)$ holding other variables constant (Table 3 ). Nevertheless, over a quarter of respondents stated they felt more depressed since the arrival of COVID-19 (29\%), and since the start of the lockdown (27\%) (Supplementary material, Table II).

\section{Correlates of Self-rated ART Adherence Score During the Lockdown}

The study secondly aimed to investigate correlates of ART adherence during the lockdown. There was no evidence of an association between adherence score and socio-demographic variables such as age, gender, education, or household income (Table 6). There was little variation in the random intercepts for ART clinics (0.11, std error 0.24); the intra-class correlation was estimated at $0.04(95 \% \mathrm{CI}$ $0.0005-0.76)$.

\section{Structural Factors}

There was limited evidence of an association between health care access and ART adherence. PLWH who missed an appointment at the health clinic because of the lockdown reported lower adherence scores $(-1.30, \mathrm{t}=-2.69$, $\mathrm{p}=0.009)$, which weakened after controlling for sociodemographic variables $(-1.13, \mathrm{t}=-2.04, \mathrm{p}=0.05)$ (Table 7). There was evidence that PLWH who ran out of medication due to the lockdown reported lower adherence scores $(-1.02, \mathrm{t}=-1.92, \mathrm{p}=0.06)$, which dropped after adjustment $(-0.92, \mathrm{t}=-1.52, \mathrm{p}=0.13)$.

\section{Social (Household) Factors}

The analysis returned further evidence of the link between household environments and ART adherence. Importantly, PLWH who stated that in their household adult food consumption was often restricted in order to feed young children also reported lower adherence scores $(-1.19, \mathrm{z}=-2.33$, $\mathrm{p}=0.02$ ), holding other variables constant (Table 7).

There was fairly strong evidence that experiencing all forms of violence was associated with lower adherence scores in bivariate and multivariate analysis (Table 7) (e.g. any reported violence: $-2.09, \mathrm{t}=-2.55,=0.01)$, as well as

Table 6 Crude and adjusted socio-demographic correlates of self-reported adherence score

\begin{tabular}{|c|c|c|c|c|c|c|}
\hline Variable* & Bivariate estimate & t \& p-value & $95 \% \mathrm{CI}$ & $\begin{array}{l}\text { Adjusted } \\
\text { estimate* }\end{array}$ & t \& p-value & $95 \% \mathrm{CI}$ \\
\hline Baseline adherence & 0.09 & $0.86, p=0.39$ & -0.11 to 0.28 & 0.08 & $0.77, p=0.44$ & -0.13 to 0.30 \\
\hline Age (centered at the mean) & 0.03 & $1.46, p=0.15$ & -0.01 to 0.07 & 0.04 & $1.67, p=0.10$ & -0.007 to 0.08 \\
\hline \multicolumn{7}{|l|}{ Gender } \\
\hline Female & (base) & & & & & \\
\hline Male & -0.01 & $-0.02, p=0.98$ & -0.82 to 0.80 & -0.11 & $-0.23, p=0.82$ & -1.04 to 0.82 \\
\hline \multicolumn{7}{|l|}{ Education category } \\
\hline None-/some secondary & (base) & & & & & \\
\hline Matric/degree/diploma & 0.34 & $0.85, p=0.40$ & -0.45 to 1.12 & 0.34 & $0.78, p=0.44$ & -0.54 to 1.22 \\
\hline \multicolumn{7}{|l|}{ Household income } \\
\hline $0-2000$ rand a month & (base) & & & & & \\
\hline $2000-5000$ rand $\mathrm{pcm}$ & 0.19 & $0.43, p=0.67$ & -0.70 to 1.09 & 0.13 & $0.29, p=0.78$ & -0.80 to 1.07 \\
\hline $5000-50000$ rand $\mathrm{pcm}$ & 0.45 & $0.74, p=0.46$ & -0.75 to 1.65 & 0.5 & $0.79, p=0.43$ & -0.77 to 1.77 \\
\hline \multicolumn{7}{|l|}{ Intervention } \\
\hline Control & (base) & & & (base) & & \\
\hline Intervention & 0.43 & $1.01, p=0.35$ & -0.62 to 1.48 & 0.47 & $0.95, p=0.38$ & -0.73 to 1.68 \\
\hline Cons & - & & & 7.89 & $<0.001$ & 5.75 to 10.04 \\
\hline \multicolumn{3}{|l|}{ Random effects } & \multicolumn{4}{|c|}{ SE } \\
\hline $\begin{array}{l}\text { ART clinic: var } \\
\text { (cons) }\end{array}$ & & & \multicolumn{2}{|c|}{0.11} & 0.24 & 0.001 to 8.14 \\
\hline Var (residual) & & & \multicolumn{2}{|c|}{2.86} & 0.51 & 2.01 to 4.06 \\
\hline
\end{tabular}

*Adjusted for: baseline adherence score, age, education, household income, intervention group 


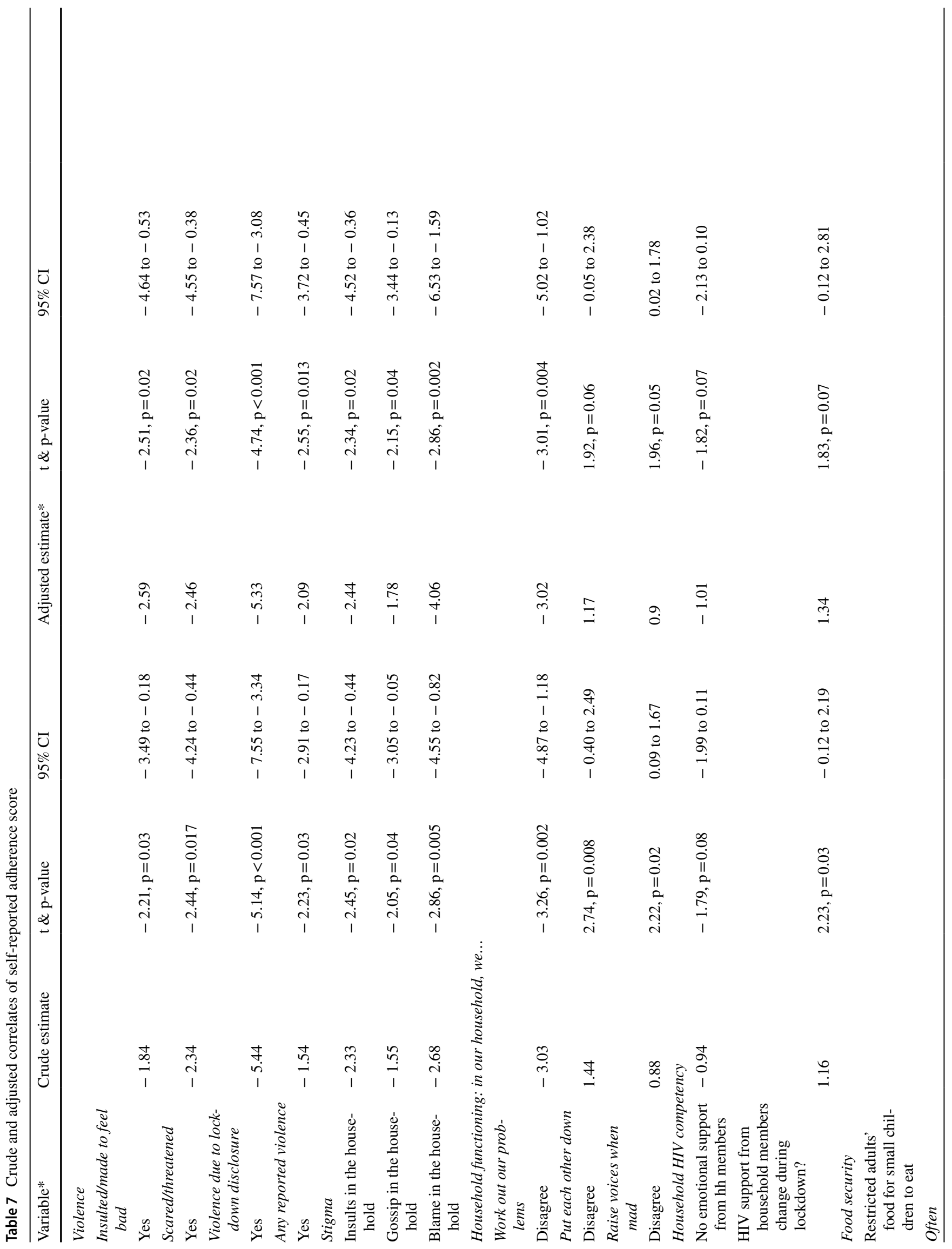


violence experienced due to disclosure of HIV status during the lockdown $(-5.33, \mathrm{t}=-4.74, \mathrm{p}<0.001)$. Experiencing stigma (insults, blame and gossip in the household) was negatively associated with adherence scores: those experiencing blame averaged 4.06 adherence points fewer than those who $\operatorname{did} \operatorname{not}(\mathrm{t}=-2.86, \mathrm{p}=0.002)$ (Table 7).

PLWH whose household members who did not work together to work out problems reported lower adherence scores $(-3.02, \mathrm{t}=-3.01, \mathrm{p}=0.004)$, and reported higher adherence scores when household members did not raise their voices when angry $(0.90, t=1.96, p=0.05)$ (Table 7). There was weak evidence that participants reported higher adherence scores when they stated that household members did not put each other down $(1.17, t=1.92, p=0.06)$ (Table 7).

\section{Individual Factors: Self-reported Well-Being and Depression}

Those reporting feeling more depressed during the lockdown also reported lower adherence scores $(-1.17, \mathrm{t}=-2.47$, $\mathrm{p}=0.02$ ), holding all other variables constant (Table 7). The pathways between depression and ART adherence were, however, complex. Those describing depression also recounted worse well-being $(-7.43, \mathrm{p}=0.031)$. Higher wellbeing scores were associated with better adherence scores $(0.43, \mathrm{t}=2.38, \mathrm{p}=0.02)$ although this association weakened when controlling for socio-demographic variables $(0.39$, $\mathrm{t}=1.81, \mathrm{p}=0.08)$ (Table 7). Participants also had lower odds of reporting depression with indicators of improved household functioning (household members don't raise voices when angry: $\mathrm{aOR}: 0.27, \mathrm{z}=-2.04, \mathrm{p}=0.04$ ), which was also associated with better adherence scores.

\section{Discussion}

This study examined data from PLWH in Cape Town, focusing on two objectives: first, assessing differences (e.g., in household income) between baseline and follow-up in individual, social (household) and structural factors that affect ART adherence; second, examining the correlates of ART adherence at follow-up. It was hypothesized that lockdown would negatively impact well-being, household functioning, food security and access to care, and that these would be associated with ART adherence at follow-up. The results indicated that the impact of lockdown is unequal, dependent on a complex web of individual, social and structural factors.

While almost a third of participants reported feeling more depressed during the lockdown, there was also surprising evidence of an improvement in individual well-being, in contrast to studies in Uganda and New Zealand [29, 47]. Better mental health was, however, linked to more supportive 
household environments. Whilst an increase in household tension was predicted in South Africa [48], this study found evidence of some improvement at follow-up in indicators for household functioning, household HIV competency and household HIV stigma-in contrast to the initial hypothesis. Similarly, a study from Spain also found evidence of relational improvement after the lockdown, although this was dependent on parental status [49]. A large body of research demonstrates the importance of connections and social support for well-being [50-52]; social support and close relationships with household members can generate resilience and offset stressors [32, 33, 53]. Households with better functioning and supportive environments were therefore perhaps more resilient to the multiple stressors created by the COVID-19 lockdown: the lockdown may have created a focus and/or space for feelings of togetherness and thereby strengthened relationships in these households [49], while the mental health of patients with less supportive households suffered. It is therefore unsurprising that interlinked individual and household factors were associated with ART adherence, and that the impact of the lockdown on PLWH differentiated accordingly, with improvements for some and deterioration for others. Further qualitative research would be helpful in illuminating changes brought about in households as a result of the lockdown.

The association between IPV and adherence exemplifies the link between household environments and individual health behaviour. Reported IPV during the lockdown was strongly associated with worse adherence to ART. Joska et al. (2020) underlined that engaging with HIV services during the lockdown may even increase risk of violence in the home for PLWH [30]: adherence may therefore be compromised for self-protection. In contradiction to the initial hypothesis, this study found a reduction in the odds of reporting IPV during the lockdown, which is in accordance with South Africa's official crime statistics, as well as reports of hospital admissions due to assault and sexual assault [54-56] though other sources have cited increases in IPV during the lockdown in South Africa [30, 57]. Globally, a large number of studies have reported increased rates of IPV during lockdown measures [58-67], while a smaller number have reported decreases in overall IPV, assistance-seeking, or changes in types of IPV [60, 65, 68-72]. Reductions in IPV were hoped for when the alcohol sales ban in South Africa was implemented during the lockdown [12]: one study indicated that deaths from "unnatural causes" decreased dramatically during the lockdown [73] while another links a reduction in hospital admissions for assault and sexual assault directly to the alcohol ban [56]. Conversely, qualitative research has presented the ban as a stressor and therefore a cause of IPV [74], warranting further research on the link between the alcohol ban and IPV. Interestingly, participants in this study who reported violence both at baseline and at follow-up had specified at baseline that the perpetrator was their current partner or household member living in the household: reductions in violence could therefore be due to reduced contact with perpetrators who live outside the household. Levels of violence were furthermore expected to rise again once measures eased [75]. Results for this study may also, of course, be affected by under-reporting, especially if a perpetrator is in the room or building.

As lockdown resulted in a loss of employment for many in South Africa, there has been much discussion around the impact on household income, especially for the most vulnerable [4, 48, 76-81]. Between baseline and followup there was, as hypothesized, evidence of a decrease in the odds of being in a higher household income category, and an increase in the odds of falling below the poverty line, a finding in line with government reports, quantitative studies, and modelling studies in South Africa [79-84]. Although lower-income households in South Africa may have been somewhat protected from the economic shock of the pandemic due to cash transfers from the government [85], implementation and uptake has been uneven: many households have still suffered a loss of income. No evidence was found for an association between income and ART adherence, although measuring household income in a sufficient degree of granularity is difficult, particularly when interviewing only one individual per household [86] — and household income alone does not effectively represent socio-economic status [87]. Similarly, no evidence was found for an association between the education level of the respondent and ART adherence. This is in contrast to other studies in South Africa [88-90], however, assessing the relationship between ART adherence and factors such as education is notoriously complex given the multiple pathways between them [36, 91], especially in times of crisis.

One concern regarding loss of employment and informal income sources during lockdown revolved around the impact on household food security, as a result of exacerbated structural food and economic insecurity impacting the ability of households to procure enough food [29, 92, 93]. In this study, those who restricted adults' consumption so that young children could eat during lockdown reported worse adherence. Prior to school closures during the pandemic, households may have benefitted from children eating meals at school [48]: although the South African government increased the amount of the child support grants during the lockdown [94], this was calculated per-caregiver, not perchild, thereby penalizing those with larger families. Grants should therefore be raised on a per-child basis: caregiver ART adherence may depend on it.

There was limited evidence that structural factors such as healthcare access were important for ART adherence. 
Whilst missing an appointment during lockdown was associated with lower adherence scores, participants also reported issues with accessing medication and missing the support offered by the CHW. Similarly, the National Income Dynamics Study, South Africa's Coronavirus Rapid Mobile Survey (NIDS-CRAM), also reported a reduction in visits for HIV care during the lockdown [95]. Although facilities were open throughout the lockdown, access to care may have been affected by a number of factors: fear of contracting COVID-19 when attending health facilities, reduced income and transport issues, as well as facility closures due to outbreaks and/or isolating staff members [13]. Access also varies by location: studies in KwaZulu Natal found no evidence of a reduction in clinic visits, although COVID-19 regulations were noticeably less strictly enforced in rural areas $[13,96]$. Dorward et al. posited that an increase in ART collections prior to lockdown may have reflected stockpiling in anticipation of restricted access to health care [13]. Qualitative research should analyse patient strategies for accessing health care prior to and during the lockdown in order to inform policy for potential future outbreaks.

\section{Limitations}

This study is subject to a number of limitations. First, the number of clusters (ART clinics) is small when compared to recommendations in the literature [97, 98]: assessing variability at the district level is therefore challenging. The intra-class correlation coefficients for adherence were small $(<0.05)$; however, ignoring cluster design in sensitivity analyses gave lower p-values, indicating the importance of accounting for clustering. There is a pressing need for methodological studies expanding understanding of mixed effects modelling with few clusters and small sample sizes, especially with binary outcomes.

Second, of 152 baseline participants, only 83 participated in the follow-up, which could introduce selection bias. Participants had to be contacted via telephone to adhere to COVID regulations: this was challenging given the necessity of participants being able to charge batteries. Restricted maximum likelihood was therefore used with a Kenward-Roger correction in order to account for the small sample [43], and the recommendations of Dinh et al. followed for handling missing data [44]. For binary outcomes, as the h-likelihood was not available in Stata, Gauss-Hermite quadrature was chosen as the Laplacian approximation has been known to produce biased parameter estimates; even though the bias is more conspicuous in the variance components estimates.

Third, there was evidence of a difference in terms of education level between baseline and those who followed up to complete the second questionnaire: those with higher levels of education had higher odds of completing the follow-up questionnaire. One possible explanation could be that those with higher levels of education may have continued working during the lockdown [80, 85]. A report for the Coronavirus Rapid Mobile Survey undertaken in South Africa states that those with higher education levels were more likely to be in formal jobs such as managers, professionals, technicians, whereas those with lower levels of education were more likely to be in informal work, e.g. craft and trade, plant and machine operators [79]. Informal jobs were more vulnerable to being lost and suffering higher wage losses [79, 80, 99]. Those with higher levels of education may therefore have been available to participate in the follow-up questionnaire as they had resources available (phone, economic security and time). This could be evaluated with further qualitative research.

The potential impact of the loss to follow-up on this study's results, however, is important to address. Studies in South Africa have indicated an association between higher education level and decreased likelihood of perceived household and community HIV stigma [100-102]. Regarding IPV, systematic reviews of the literature have indicated that the link between education level and IPV is unclear [103, 104], although a meta-analysis of studies in sub-Saharan Africa has found a link between lower levels of education and higher prevalence of IPV [104]. Our results should therefore be read in light of the fact that there was a difference in the education level of those who responded to the follow-up questionnaire and those who were lost to follow-up; further quantitative and qualitative research is therefore necessary to gain a clearer picture of the impact of COVID-19 measures on IPV and HIV stigma within the home and the community.

Fourth, the main outcome was a self-reported adherence score, which may be subject to social desirability bias [105]. Although there is no gold standard for measuring adherence [106], additional analysis should be undertaken using other adherence measures to assess these findings. Additionally, the time periods for certain variables were not equal: for example, whereas baseline participants were asked about incidents of violence during the previous six months, follow-up participants were questioned about the lockdown period (about three months' duration), which is shorter: any decrease may therefore be due to differing time periods. Furthermore, given that this study was undertaken only three-four months after the beginning of the lockdown period, any changes may be temporary and in response to an urgent crisis situation: further research should analyse whether changes have endured. Nevertheless, this study provides a unique insight into the impact of the lockdown on ART patients. 


\section{Conclusions}

Implementing lockdowns to curb COVID-19 transmission resulted in a reorganization of social and economic life in South Africa on an exceptional scale, impacting ART adherence via multiple interconnected pathways. The results of this study indicate the importance of household level factors in ART adherence during lockdown, as well as individual and structural level factors. In order to support ART adherence during lockdown, the South African government should facilitate access to food and medication, especially for households with children, as well as access to IPV support services. Qualitative research should be undertaken to clarify the pathways by which individual, social and structural factors are linked to ART adherence during lockdown. Further research should assess whether interventions that improve household functioning and household HIV competency enable PLWH to adhere to ART-even in times of crisis.

Supplementary Information The online version contains supplementary material available at https://doi.org/10.1007/s10461-021-03541-0.

Acknowledgements We wish to thank Mulalo Kenneth Muhali and Jessicah Mvelase for their fieldwork supervision and intervention coordination, as well as the field workers who conducted the survey interviews, especially during the lockdown period in South Africa.

Author Contributions LC was responsible for statistical analysis and interpretation of data, and drafting and revising the manuscript. EW, CM and LC developed the manuscript concept. LK managed the trial, oversaw data acquisition, and supervised fieldwork teams. EW, LK and $\mathrm{CM}$ were responsible for SINAKO study design and acquisition of funding. All authors provided critical revision of the article, and read and approved the final version of the manuscript.

Funding VLIR-UOS Research Foundation-Flanders awarded this TEAM initiative. VLIR-UOS reference number: ZA2018TEA474A102. Research Foundation Flanders awarded project funding under Grant G035018N, as well as post-doctoral fellowship $12 \mathrm{~T} 8119 \mathrm{~N}$. The National Research Foundation, South Africa: awarded the study a Research Development Grant for Y-Rated Researchers (grant number 116356). The funding bodies had no role in the design of the study, in the collection, analysis or interpretation of data, in writing the manuscript or in deciding to submit the manuscript for publication.

Availability of Data and Material The datasets analyzed during the current study are available from the corresponding author on reasonable request.

Code Availability The coding used during the current study is available from the corresponding author on reasonable request.

\section{Declarations}

Competing interests The authors declare no competing interests.

Ethics Approval The study was approved by the ethics committee of the University of the Western Cape (BM19/4/6, June 2019) and the ethical committee for the Social Sciences and Humanities of the University of Antwerp (SHW_17_64, September 2018). The City of Cape Town and the Western Cape Department of Health granted permission for all facilities by December 2019. Ethical approval was updated for the follow-up interviews (SHW_17_64 (wijziging), BM19/4/6, August 2020). All participants provided written informed consent at baseline in their chosen language, and oral informed consent during the followup questionnaire.

Consent to Participate Informed consent was obtained from all individual participants included in the study.

Consent for Publication Patients signed informed consent regarding publishing their anonymized data.

\section{References}

1. European Council CotEU. Timeline - Council actions on COVID-19 2020. https://www.consilium.europa.eu/en/policies/ coronavirus/timeline/\#.

2. WHO. Timeline of WHO's response to COVID-19. 2020.

3. Hargreaves J, Davey C, Hargreaves J, et al. Three lessons for the COVID-19 response from pandemic HIV. Lancet HIV. 2020;7(5):e309-11.

4. Hamadani JD, Hasan MI, Baldi AJ, et al. Immediate impact of stay-at-home orders to control COVID-19 transmission on socioeconomic conditions, food insecurity, mental health, and intimate partner violence in Bangladeshi women and their families: an interrupted time series. Lancet Glob Health. 2020;8(11):e1380-9.

5. Hara M, Ncube B, Sibanda D. Water and sanitation in the face of Covid-19 in Cape Town's townships and informal settlements 2020. https://www.plaas.org.za/water-and-sanitation-in-the-faceof-covid-19-in-cape-towns-townships-and-informal-settlements/.

6. Kelley M, Ferrand RA, Muraya K, et al. An appeal for practical social justice in the COVID-19 global response in lowincome and middle-income countries. Lancet Glob Health. 2020;8(7):e888-9.

7. Nyoni T, Okumu M. COVID-19-compliant strategies for supporting treatment adherence among people living with HIV in Sub-Saharan Africa. AIDS Behav. 2020;24(9):2473-6.

8. Diseases NIfC. First case of COVID-19 coronavirus reported in SA South Africa 2020. https://www.nicd.ac.za/first-caseof-covid-19-coronavirus-reported-in-sa/\#: :text=This\%20mor ning $\% 2$ C $\% 20$ Thursday $\% 20$ March $\% 205$,Africa\%20on\%20Mar ch $\% 201 \% 2 \mathrm{C} \% 202020$.

9. Zuma DND. Declaration of a National State of Disaster. In: Affairs DoC-OGaT, editor. South Africa 2020.

10. Bauer N. Level 5 to 4: South Africa plans to reopen economy, in phases. Al Jazeera. 2020;2020:24.

11. van Dyk J. Will Ramaphosa announce an end to South Africa's national alcohol ban today? Bhekisisa. 2020.

12. Matzopoulos R, Walls H, Cook S, et al. South Africa's COVID19 Alcohol Sales Ban: the potential for better policy-making. Int J Health Policy Manag. 2020;9(11):486-7.

13. Dorward J, Khubone T, Gate K, et al. The impact of the COVID-19 lockdown on HIV care in 65 South African primary care clinics: an interrupted time series analysis. Lancet HIV. 2021;8(3):e158-65.

14. Mendelsohn AS, Ritchwood T. COVID-19 and antiretroviral therapies: South Africa's charge towards 90-90-90 in the midst of a second pandemic. AIDS Behav. 2020;24(10):2754-6. 
15. Zhou F, Yu T, Du R, et al. Clinical course and risk factors for mortality of adult inpatients with COVID-19 in Wuhan, China: a retrospective cohort study. Lancet. 2020;395(10229):1054-62.

16. Simbayi L, Zuma K, Zungu N, et al. South African national HIV prevalence, incidence, behaviour and communication survey, 2017: towards achieving the UNAIDS 90-90-90 targets. 2019.

17. Bank W. Poverty \& Equity Brief, South Africa 2020. https:// databank. worldbank.org/data/download/poverty/33EF03BB9722-4AE2-ABC7-AA2972D68AFE/Global_POVEQ_ZAF.pdf.

18. Boulle A, Davies M-A, Hussey H, et al. Risk factors for COVID19 death in a population cohort study from the Western Cape Province, South Africa. Clin Infect Dis. 2020:ciaa1198.

19. Attia S, Egger M, Müller M, et al. Sexual transmission of HIV according to viral load and antiretroviral therapy: systematic review and meta-analysis. AIDS. 2009;23(11):1397-404.

20. Cohen MS, Chen YQ, McCauley M, et al. Prevention of HIV-1 infection with early antiretroviral therapy. N Engl J Med. 2011;365(6):493-505.

21. Taylor G. Rolling out HIV antiretroviral therapy in sub-Saharan Africa: 2003-2017. Can Commun Dis Rep. 2018;44(2):68-70.

22. WHO. Consolidated Guidelines on the Use of Antiretroviral Drugs for Treating and Preventing HIV Infection,. Recommendations for a Public Health Approach. Geneva: World Health Organisation; 2016. p. 2016.

23. Jewell BL, Mudimu E, Stover J, et al. Potential effects of disruption to HIV programmes in sub-Saharan Africa caused by COVID-19: results from multiple mathematical models. Lancet HIV. 2020;7(9):e629-40.

24. Shiau S, Krause KD, Valera P, et al. The burden of COVID-19 in people living with HIV: a syndemic perspective. AIDS Behav. 2020;24(8):2244-9.

25. Hogan AB, Jewell BL, Sherrard-Smith E, et al. Potential impact of the COVID-19 pandemic on HIV, tuberculosis, and malaria in low-income and middle-income countries: a modelling study. Lancet Glob Health. 2020;8(9):e1132-41.

26. Amimo F, Lambert B, Magit A, et al. The potential impact of the COVID-19 pandemic on HIV, tuberculosis, and malaria control in Africa: a systematic review of modelling studies and population surveys. Research Square; 2020.

27. Bradbury-Jones C, Isham L. The pandemic paradox: the consequences of COVID-19 on domestic violence. J Clin Nurs. 2020;29(13-14):2047-9.

28. Johnson K, Green L, Volpellier M, et al. The impact of COVID19 on services for people affected by sexual and gender-based violence. Int J Gynecol Obstet. 2020;150(3):285-7.

29. Mahmud M, Riley E. Household response to an extreme shock: Evidence on the immediate impact of the Covid-19 lockdown on economic outcomes and well-being in rural Uganda. World Development. 2021;140:105318.

30. Joska JA, Andersen L, Rabie S, et al. COVID-19: increased risk to the mental health and safety of women living with HIV in South Africa. AIDS Behav. 2020;24(10):2751-3.

31. Masquillier C, Knight L, Campbell L, et al. Sinako, a study on HIV competent households in South Africa: a cluster-randomised controlled trial protocol. Trials. 2020;21(1):154

32. Masquillier C, Wouters E, Mortelmans D, et al. On the road to HIV/AIDS competence in the household: building a healthenabling environment for people living with HIV/AIDS. Int J Environ Res Public Health. 2015;12(3):3264-92.

33. Masquillier C, Wouters E, Mortelmans D, et al. HIV/AIDS competent households: interaction between a health-enabling environment and community-based treatment adherence support for people living with HIV/AIDS in South Africa. PLoS ONE. 2016;11(3):379.

34. Wouters E. Life with HIV as a chronic illness: a theoretical and methodological framework for antiretroviral treatment studies in resource-limited settings. Soc Theory Health. 2012;10(4):368-91.

35. Wouters E, Masquillier C, Ponnet K, et al. A peer adherence support intervention to improve the antiretroviral treatment outcomes of HIV patients in South Africa: the moderating role of family dynamics. Soc Sci Med. 2014;113:145-53.

36. Campbell L, Masquillier C, Thunnissen E, et al. Social and structural determinants of household support for ART adherence in low- and middle-income countries: a systematic review. Int J Environ Res Public Health. 2020;17(11):3808.

37. Ruch W. National Poverty Lines 2020. In: Africa SS, editor. 2020.

38. Shisana O, Labadarios D, Rehle T, et al. The South African National Health and Nutrition Examination Survey, 2012: SANHANES-1: the health and nutritional status of the nation. Human Sciences Research Council; 2014.

39. Garcia-Moreno C, Jansen HAFM, Ellsberg M, et al. Prevalence of intimate partner violence: findings from the WHO multicountry study on women's health and domestic violence. Lancet. 2006;368(9543):1260-9.

40. Council HSR. The people living with HIV stigma index: An index to measure the stigma and discrimination experienced by people living with HIV in South Africa. 2014.

41. Fok CCT, Allen J, Henry D, et al. The brief family relationship scale: a brief measure of the relationship dimension in family functioning. Assessment. 2014;21(1):67-72.

42. Donner A, Klar N. Design and analysis of cluster randomization trials in health research. London: Arnold; 2010.

43. Dinh P, Yang P. Handling baselines in repeated measures analyses with missing data at random. J Biopharm Stat. 2011;21(2):326-41.

44. Elff M, Heisig JP, Schaeffer M, et al. Multilevel analysis with few clusters: improving likelihood-based methods to provide unbiased estimates and accurate inference. Br J Polit Sci. 2021;51(1):412-26.

45. McNeish D. Small sample methods for multilevel modeling: a colloquial elucidation of REML and the Kenward-Roger correction. Multivar Behav Res. 2017;52(5):661-70.

46. Paramesha AE, Chacko LK. Predictors of adherence to antiretroviral therapy among PLHIV. Indian J Public Health. 2019;63(4):367-76

47. Sibley CG, Greaves LM, Satherley N, et al. Effects of the COVID-19 pandemic and nationwide lockdown on trust, attitudes toward government, and well-being. Am Psychol. 2020;75(5):618-30.

48. Moore E. Family dynamics in multi-generational households during Covid-19 2020. https://www.dailymaverick.co.za/artic le/2020-04-17-family-dynamics-in-multi-generational-house holds-during-covid-19/.

49. Günther-Bel C, Vilaregut A, Carratala E, et al. A mixedmethod study of individual, couple, and parental functioning during the state-regulated COVID-19 lockdown in Spain. Fam Process. 2020;59(3):1060-79.

50. Holt-Lunstad J, Smith TB, Baker M, et al. Loneliness and social isolation as risk factors for mortality: a meta-analytic review. Perspect Psychol Sci. 2015;10(2):227-37.

51. Jetten J, Haslam C, Haslam SA. The social cure: Identity, health and well-being. In: Jetten J, Haslam C, Haslam SA, editors. New York: Psychology Press; 2012

52. Valtorta NK, Kanaan M, Gilbody S, et al. Loneliness and social isolation as risk factors for coronary heart disease and stroke: systematic review and meta-analysis of longitudinal observational studies. Heart. 2016;102(13):1009-16.

53. Gard AM, McLoyd VC, Mitchell C, et al. Evaluation of a longitudinal family stress model in a population-based cohort. Soc Dev. 2020;29(4):1155-75. 
54. Studies IfS. SA crime reductions during COVID-19 lockdown may be short lived 2020. https://issafrica.org/about-us/pressreleases/sa-crime-reductions-during-covid-19-lockdown-maybe-short-lived\#: :text=Lockdown\%20had\%20an\%20effect $\%$ 2C\%20but $\% 20$ not $\% 20$ everywhere \& text $=$ For $\% 20$ example $\%$ 2C\%20in\%20April\%20total,crime\%20was\%20far\%20less\% 20 pronounced.

55. Stiegler N, Bouchard J-P. South Africa: challenges and successes of the COVID-19 lockdown. Annales Médico-psychologiques, revue psychiatrique. 2020;178(7):695-8.

56. Reuter H, Jenkins LS, De Jong M, et al. Prohibiting alcohol sales during the coronavirus disease 2019 pandemic has positive effects on health services in South Africa. Afr J Prim Health Care Fam Med. 2020;12(1):e1-4.

57. Nduna M, Domesticated TSO, During P-V the Covid-19 lockdown in South Africa. Psychol Stud (Mysore). 2020;2021:1-7.

58. Agüero JM. COVID-19 and the rise of intimate partner violence. World Dev. 2021;137:105217.

59. Fawole OI, Okedare OO, Reed E. Home was not a safe haven: women's experiences of intimate partner violence during the COVID-19 lockdown in Nigeria. BMC Womens Health. 2021;21(1):32.

60. Gosangi B, Park H, Thomas R, et al. Exacerbation of physical intimate partner violence during COVID-19 pandemic. Radiology. 2021;298(1):E38-45.

61. Hsu L-C, Henke A. COVID-19, staying at home, and domestic violence. Rev Econ Household. 2021;19(1):145-55.

62. Mahmood KI, Shabu SA, KM MA, et al. The impact of COVID19 related lockdown on the prevalence of spousal violence against women in Kurdistan region of Iraq. J Interpers Violence. 2021:886260521997929.

63. Sediri S, Zgueb Y, Ouanes S, et al. Women's mental health: acute impact of COVID-19 pandemic on domestic violence. Arch Womens Ment Health. 2020;23(6):749-56.

64. Hamadani JD, Hasan MI, Baldi AJ, et al. Immediate impact of stay-at-home orders to control COVID-19 transmission on socioeconomic conditions, food insecurity, mental health, and intimate partner violence in Bangladeshi women and their families: an interrupted time series. Lancet Glob Health. 2020;8(11):e1380-9.

65. Ojeahere MI, Kumswa SK, Adiukwu F, et al. Intimate partner violence and its mental health implications amid COVID-19 lockdown: findings among Nigerian couples. J Interpers Violence. 2021:8862605211015213.

66. Rhodes HX, Petersen K, Lunsford L, et al. COVID-19 resilience for survival: occurrence of domestic violence during lockdown at a Rural American College of Surgeons verified level one trauma center. Cureus. 2020;12(8):10059.

67. Sabri B, Hartley M, Saha J, et al. Effect of COVID-19 pandemic on women's health and safety: a study of immigrant survivors of intimate partner violence. Health Care Women Int. 2020;41(11-12):1294-312.

68. Erausquin JT, Tan RKJ, Uhlich M, et al. The International Sexual Health and Reproductive Health Survey (I-SHARE-1): a cross-sectional multi-country analysis of adults from 30 countries prior to and during the initial COVID-19 wave. medRxiv. 2021:2021.09.18.21263630

69. Barbara G, Facchin F, Micci L, et al. COVID-19, lockdown, and intimate partner violence: some data from an Italian service and suggestions for future approaches. J Womens Health (Larchmt). 2020;29(10):1239-42.

70. Holland KM, Jones C, Vivolo-Kantor AM, et al. Trends in US emergency department visits for mental health, overdose, and violence outcomes before and during the COVID-19 pandemic. JAMA Psychiat. 2021;78(4):372-9.
71. Muldoon KA, Denize KM, Talarico R, et al. COVID-19 pandemic and violence: rising risks and decreasing urgent careseeking for sexual assault and domestic violence survivors. BMC Med. 2021;19(1):20.

72. Ravindran S, Shah M. Unintended consequences of lockdowns: Covid-19 and the shadow pandemic. MedRN: interdisciplinary coronavirus \& infectious disease related research (Topic). 2020.

73. Moultrie TA, Dorrington RE, Laubscher R, et al. Unnatural deaths, alcohol bans and curfews: evidence from a quasi-natural experiment during COVID-19 2021.

74. Dekel B, Abrahams N. "I will rather be killed by corona than by him...": experiences of abused women seeking shelter during South Africa's COVID-19 lockdown. PLoS ONE. 2021;16(10):e0259275.

75. Crime UNOoDa. Research brief: what crime and helpline data say about the impact of the COVID-19 pandemic on reported violence against women and girls. 2020.

76. Burke J. South African police fire rubber bullets at shoppers amid lockdown. The Guardian. 2020.

77. Conversation T. COVID-19: how the lockdown has affected the health of the poor in South Africa. South Africa; 2020.

78. Council A. COVID-19 pandemic: in a nation of extreme inequality, South Africa's poorest are most at risk 2020. https:// www.atlanticcouncil.org/blogs/africasource/covid-19-pande mic-in-a-nation-of-extreme-inequality-south-africas-poore st-are-most-at-risk/.

79. Benhura M, Magejo P. Differences between formal and informal workers' outcomes during the COVID-19 crisis lockdown in South Africa. South Africa: National Income Dynamics Study (NIDS); 2020.

80. Jain R, Budlender J, Zizzamia R, et al. The labor market and poverty impacts of COVID-19 in South Africa.

81. Ranchhod V, Daniels RC. Labour market dynamics in South Africa at the onset of the COVID-19 pandemic. S Afr J Econ. 2021;89(1):44-62.

82. Africa DoSS. Loss of income resulting from the COVID-19 pandemic may lead to higher levels of food insecurity SA. 2020.

83. Arndt C, Davies R, Gabriel S, et al. Covid-19 lockdowns, income distribution, and food security: an analysis for South Africa. Global Food Security. 2020;26:100410.

84. Chitiga-Mabugu M, Henseler M, Mabugu R, et al. Economic and distributional impact of COVID-19: evidence from macromicro modelling of the South African economy. S Afr J Econ. 2020;89:82.

85. Arndt C, Davies R, Gabriel S, et al. Covid-19 lockdowns, income distribution, and food security: an analysis for South Africa Global Food Security. 2020;26:100410.

86. Sweeney S, Vassall A, Foster N, et al. Methodological issues to consider when collecting data to estimate poverty impact in economic evaluations in low-income and middle-income countries. Health Econ. 2016;25:42-52.

87. Howe LD, Galobardes B, Matijasevich A, et al. Measuring socioeconomic position for epidemiological studies in low- and middle-income countries: a methods of measurement in epidemiology paper. Int J Epidemiol. 2012;41(3):871-86.

88. Haberer JE, Bwana BM, Orrell C, et al. ART adherence and viral suppression are high among most non-pregnant individuals with early-stage, asymptomatic HIV infection: an observational study from Uganda and South Africa. J Int AIDS Soc. 2019;22(2):25232.

89. Adeniyi OV, Ajayi AI, Ter Goon D, et al. Factors affecting adherence to antiretroviral therapy among pregnant women in the Eastern Cape, South Africa. BMC Infect Dis. 2018;18(1):175.

90. Barry A, Ford N, El-Khatib Z. Factors for incomplete adherence to antiretroviral therapy including drug refill and clinic visits 
among older adults living with human immunodeficiency viruscross-sectional study in South Africa. Trop Med Int Health. 2018;23(3):270-8.

91. Peltzer K, Pengpid S. Socioeconomic factors in adherence to HIV therapy in low- and middle-income countries. J Health Popul Nutr. 2013;31(2):150-70.

92. Kalichman SC, Eaton LA, Berman M, et al. Intersecting pandemics: impact of SARS-CoV-2 (COVID-19) protective behaviors on people living with HIV, Atlanta, Georgia. J Acquir Immune Defic Syndr. 2020;85(1):66-72.

93. Aristide C, Okello S, Bwana M, et al. Learning from people with HIV: their insights are critical to our response to the intersecting COVID-19 and HIV pandemics in Africa. AIDS Behav. 2020;24(12):3295-8.

94. Webb C, Vally N. South Africa has raised social grants: why this shouldn't be a stop-gap measure. The Conversation; 2020.

95. Burger R, Day C, Deghaye N, et al. Examining the unintended consequences of the COVID-19 pandemic on public sector health facility visits: the first 150 days. 2020.

96. Siedner MJ, Kraemer JD, Meyer MJ, et al. Access to primary healthcare during lockdown measures for COVID-19 in rural South Africa: an interrupted time series analysis. BMJ Open. 2020;10(10):43-763.

97. Donner A, Klar N. Pitfalls of and controversies in cluster randomization trials. Am J Public Health. 2004;94(3):416-22.

98. Paterson L, Goldstein H. New Statistical Methods for Analysing Social Structures: an introduction to multilevel models. Br Edu Res J. 1991;17(4):387-93.

99. Coetzee BJ, Kagee A. Structural barriers to adhering to health behaviours in the context of the COVID-19 crisis: considerations for low- and middle-income countries. Glob Public Health. 2020;15(8):1093-102.
100. Brown DC, BeLue R, Airhihenbuwa CO. HIV and AIDS-related stigma in the context of family support and race in South Africa. Ethn Health. 2010;15(5):441-58.

101. Chao L-W, Gow J, Akintola G, et al. HIV/AIDS stigma attitudes among educators in KwaZulu-Natal, South Africa. J Sch Health. 2010;80(11):561-9.

102. Visser M, Sipsma H. The experience of HIV-related stigma in South Africa. In: Liamputtong P, editor. Stigma, discrimination and living with HIV/AIDS: a cross-cultural perspective. Dordrecht: Springer; 2013. p. 205-27.

103. Tenkorang EY, Asamoah-Boaheng M, Owusu AY. Intimate partner violence (IPV) against HIV-positive women in Sub-Saharan Africa: a mixed-method systematic review and meta-analysis. Trauma, Violence, \& Abuse. 0(0):1524838020906560.

104. Nabaggala MS, Reddy T, Manda S. Effects of rural-urban residence and education on intimate partner violence among women in Sub-Saharan Africa: a meta-analysis of health survey data. BMC Womens Health. 2021;21(1):149.

105. Nieuwkerk PT, de Boer-van der Kolk IM, Prins JM, et al. Selfreported adherence is more predictive of virological treatment response among patients with a lower tendency towards socially desirable responding. Antivir Ther. 2010;15(6):913-916.

106. Castillo-Mancilla JR, Haberer JE. Adherence measurements in HIV: new advancements in pharmacologic methods and real-time monitoring. Curr HIV/AIDS Rep. 2018;15(1):49-59.

Publisher's Note Springer Nature remains neutral with regard to jurisdictional claims in published maps and institutional affiliations. 